\section{The source of $X$-rays and high-charged ions based on moderate power vacuum discharge with laser triggering}

\begin{abstract}
The source of X-ray radiation with the energy of quanta that may vary in the range $h v=1 \div 12 \mathrm{keV}$ was developed for studies in X-ray interaction with matter and modification of solid surfaces. It was based on a vacuum spark discharge with the laser triggering. It was shown in our experiments that there is a possibility to adjust X-ray radiation spectrum by changing the configuration of the electrode system when the energy stored in the capacitor is varied within the range of $1 \div 17 \mathrm{~J}$. A comprehensive study of X-ray imaging and quanta energy was carried out. These experiments were carried out for the case of both direct and reverse polarity of the voltage on the electrodes. Additionally, ion composition of plasma created in a laser-triggered vacuum discharge was analyzed. Highly charged ions $\mathrm{Zn}^{(+21)}, \mathrm{Cu}^{(+20)}$ and $\mathrm{Fe}^{(+18)}$ were observed.
\end{abstract}

Key words: laser-triggered vacuum discharge $\bullet$ sources of X-rays $\bullet$ ion composition of plasma

M. A. Alkhimova ${ }^{\bowtie}$, E. D. Vovchenko, A. S. Savelov Department of Plasma Physics (No. 21),

National Research University MEPhI

(Moscow Engineering Physics Institute),

31 Kashirskoe Str., 115409 Moscow, Russia,

Tel.: +7 9031989517 ,

E-mail: raiskiyAd@rambler.ru

\section{A. P. Melekhov, R. S. Ramakoti}

Department of Laser Thermonuclear Fusion (No. 69), National Research University MEPhI

(Moscow Engineering Physics Institute),

31 Kashirskoe Str., 115409 Moscow, Russia

\section{P. S. Krapiva, I. N. Moskalenko}

Federal State Unitary Enterprise All-Russian Research Institute of Automatics SC "Rosatom" Company,

Moscow 127055, Russia

Received: 15 July 2014

Accepted: 7 January 2015

\section{Introduction}

It is well known that high-current vacuum sparks (HVS) with plasma produced due to electrode erosion are sources of characteristic X-ray emission and highly charged ions [1-3]. However, this type of discharge requires a large amount of energy stored in a capacitor banks and an additional electrode for initiation of the discharge. The process of spark pinching leads to high intensity X-ray radiation, but it is spatially and temporally unstable. Because of these instabilities it is not easy to apply the high-current vacuum spark as a source of X-ray emission in practice.

In this note the plasma generated in a laser-triggered vacuum discharge of moderate power is introduced as a portable source of X-ray emission and highly charged ions. Such plasma can be a stable source of X-ray emission in which the intensity and the energy of quanta is determined by parameters of the laser triggering, the energy stored in the capacitor and the geometry of the discharge electrodes [4].

\section{Experimental set-up and results}

The facility consists of an interaction vacuum chamber (pressure $P \approx 10^{-3} \mathrm{~Pa}$, volume $V \approx 0.1 \mathrm{~m}^{3}$ ), an 


\section{TOF mass}

spectrometer

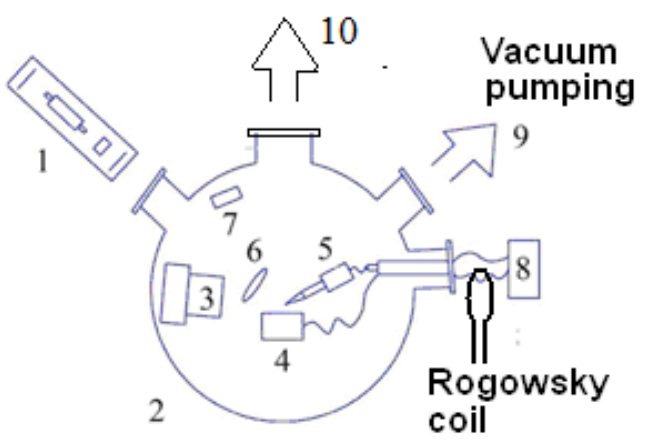

a)

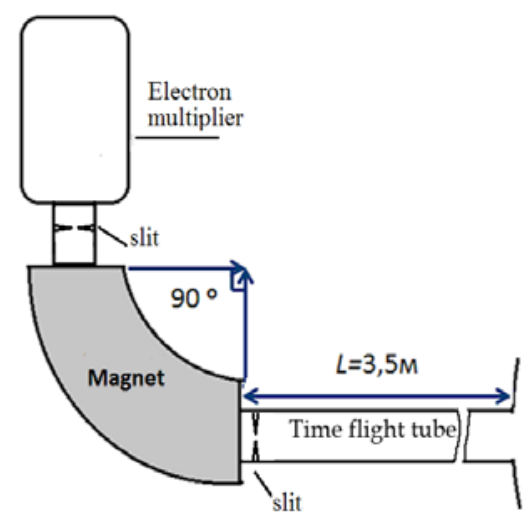

b)

Fig. 1. (a) The experimental set-up: 1 - the laser, 2 - the vacuum chamber, 3 - the X-ray pinhole camera, 4 - the target electrode, 5 - the high-voltage electrode, 6 - the focusing lens, 7 -X-ray pin-diodes, 8 - the capacitor, 9 - the vacuum pumping, 10 - the time-of-flight base; (b) the TOF mass analyzer.

Nd-YAG laser, operating at the Q-switching mode (wavelength $\lambda=1.06 \mathrm{~mm}$, pulse energy $E_{l} \approx 25 \mathrm{~mJ}$ and duration of $\tau \approx 15 \mathrm{~ns}$ ), a capacitive energy storage and diagnostic tools (the Rogowski coil, a vacuum X-ray pinhole camera with Kodak (Ultra-speed DF-58 [5]) film detector, attenuating Al filters, and X-ray pin-diodes) (Fig. 1).

To measure the temporal characteristics of the $\mathrm{X}$-ray emission and to estimate the total energy carried by X-rays per $4 \pi$ sr per shot, two identical pin-diodes were used. They were placed inside the vacuum chamber at the distance about $20 \mathrm{~cm}$ from the discharge gap, along a line perpendicular to the axis of the discharge. The input window of the first diode was covered by thin foil of $9 \mu \mathrm{m} \mathrm{Al}$ (channel 1) and the input window of the second diode was covered by thin foil of $17 \mu \mathrm{m} \mathrm{Mg}$ (channel 2). Subtraction of signals in these channels gives the energy interval of $E_{q}=1.3 \div 1.57 \mathrm{keV}$ (the range of photon energies between $\mathrm{Al} \mathrm{K-edge} \mathrm{and} \mathrm{Mg} \mathrm{K-edge).}$

To study the ion flux generated from plasma the TOF mass analyzer with a sector magnet was used. The time-of-flight (TOF) base has the length of $L=3.5 \mathrm{~m}$. The magnetic field can be changed in the range $\mathrm{H}=0 \div 0.56 \mathrm{~T}$ by varying the current in the coils of the magnet.

The discharge system consisted of electrodes, high-voltage power supply, capacitive energy storage and a low-inductance vacuum current lead. The charging voltage was varied from 5 to $13 \mathrm{kV}$. In our

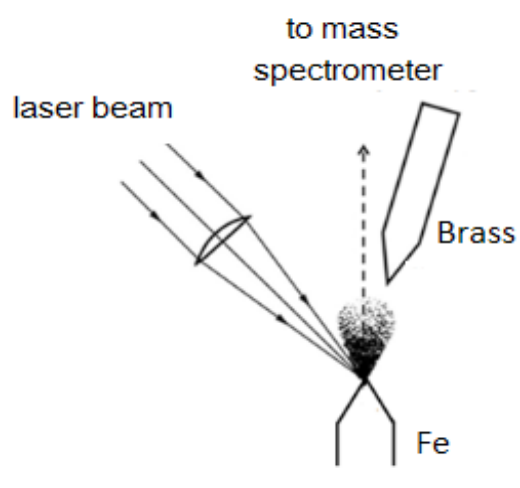

target-electrode

Fig. 2. The principal experimental scheme.

experiments we used two modes with the capacity $C_{1}=0.22 \mu \mathrm{F}$ and $C_{2}=0.011 \mu \mathrm{F}$. To increase the electric field strength and the intensity of X-ray emission electrodes of conical shape were used [6]. A laser beam is focused on the Fe electrode target (Fig. 2). This electrode is made of iron and is grounded. The other high-voltage electrode is made of brass.

The axis of the discharge is at an angle of about 30 degrees relative to the direction of ion emission into the TOF mass analyzer.

The distance between the electrodes was chosen as a variable parameter, which affects the energy range and the intensity of X-rays as well as the number of ions created in plasma. The length $d$ of the electrode gap was changed in the range $d=2 \div 7 \mathrm{~mm}$ with the step of about $1 \mathrm{~mm}$.

In the first series of our experiments we used the capacitor with $C_{1} \approx 0.22 \mu \mathrm{F}$. In this case the maximum charging voltage $U_{\max }=13 \mathrm{kV}$ corresponds the stored energy $E_{1} \sim 17 \mathrm{~J}$ and the amplitude of discharge current did not exceed the value of $I_{1} \approx 10 \mathrm{kA}$.

An X-ray image of the plasma was obtained with the vacuum pinhole camera (the diameter of the aperture $d \sim 500 \mu \mathrm{m}$, the magnification $D=1$ ). It was located inside the vacuum chamber at a distance of about $10 \mathrm{~cm}$ from the discharge plasma. The X-ray image was attenuated by means of $4 \mu \mathrm{m} \mathrm{Al}$ filters and recorded on a Kodak film.

Two modes with electrode target as a cathode and an anode were investigated. Figure 3 shows the integral (without the time resolution) spatial distribution of the X-ray emission. The highest intensity $\mathrm{X}$-rays are observed when the electrode-target is the cathode (Fig. 3a). We can see two bright regions of the X-ray radiation. Our interpretation is that the large area around the anode corresponds to the bremsstrahlung and a smaller area near the cathode shows the pinching of plasma near the cathode. When the laser pulse was directed into the anode (Fig. 3b), the bremsstrahlung emission that could be seen around the anode was weak, because the initial electrons not are accelerated in the external electric field. In all further experiments we used the electrode target as a cathode.

The measurement of the energies of the X-ray quanta was performed using the attenuating $\mathrm{Al}$-filters 
a)

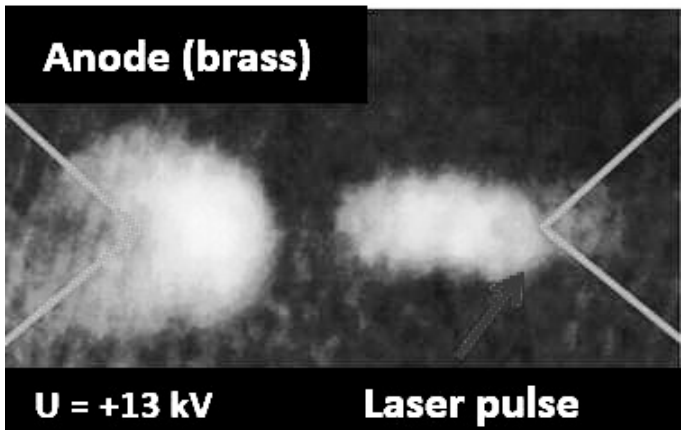

b)

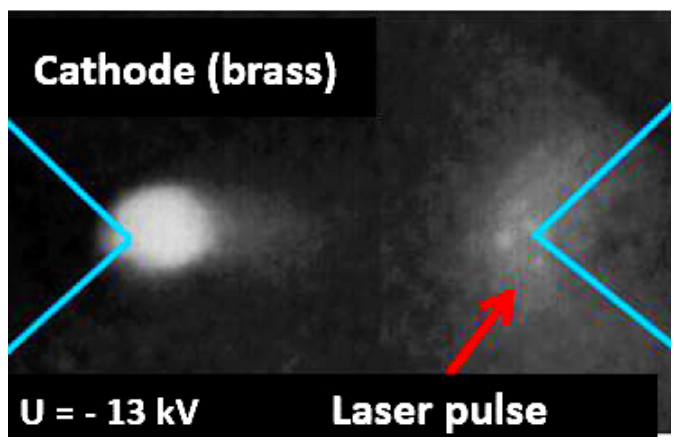

Fig. 3. X-ray images of the discharge gap for laser-triggered vacuum discharge $\left(C_{1} \approx 0.22 \mu \mathrm{F}, d=5 \mathrm{~mm}, U_{\max }=\right.$ $13 \mathrm{kV})$ : a) the electrode-target is a cathode; b) the electrode-target is an anode.

a)

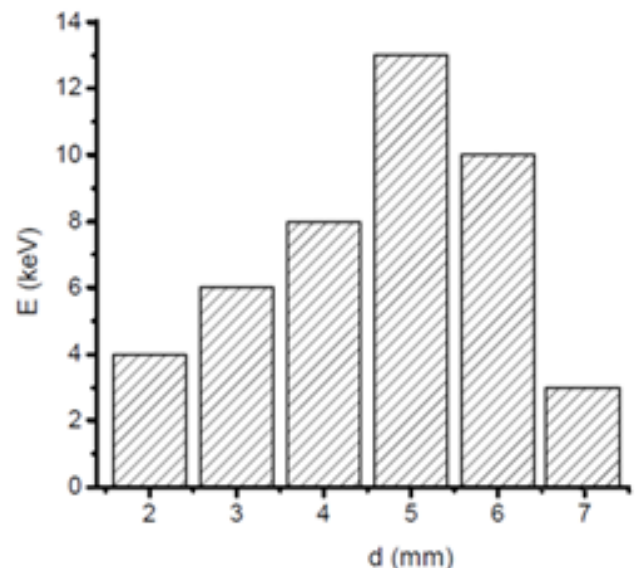

b)

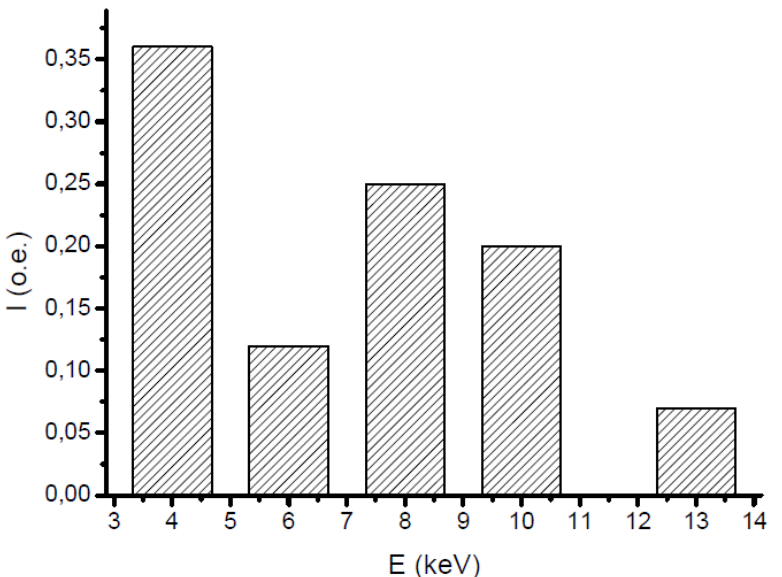

Fig. 4. Histograms of restoration of X-ray spectrum for experimental conditions $C_{1} \approx 0.22 \mu \mathrm{F}, U=-13 \mathrm{kV}, E_{1} \sim 17 \mathrm{~J}$ : a) the average photon energy of the emitted X-ray spectrum depends of the electrode gap size; $b$ ) the spectral composition of the X-ray source for the optimal gap size $d=5 \mathrm{~mm}$.

with thickness in the range $h=2 \div 120 \mu \mathrm{m}$. The cassette with $\mathrm{Al}$ filters and the Kodak film was placed at a distance of about $5 \mathrm{~cm}$ from the X-ray radiation source. Using 10 shots for each experiment we obtained the intensities of the $\mathrm{X}$-ray radiation for various thicknesses of the attenuating filter $J(h)$ :

$$
J(h)=\int S(E) \cdot \varphi(E) \cdot \exp [-\mu(E) \cdot h] \cdot d E
$$

where $S(E)$ denotes the spectral characteristics of the film, $\varphi(E)$ denotes the energy spectrum, $\mu(E)$ represents the attenuation coefficient of the X-ray radiation in matter, and $E$ is the effective energy. This expression is a Fredholm equation of the 1st kind with respect to the function $\varphi(E)$.

In our case, to recover the energy spectrum we determine the energy-dependent attenuation coefficient $\mu(E)$ of X-rays in matter by using the 'method of effective energies' previously successfully applied in the works [7-9]. The physical basis of the method is the representation of the flow of X-rays passing through the attenuation filters in the form

$$
\varphi(h)=\sum_{j=0}^{n} \varphi_{0 j} \exp \left[-\mu\left(E_{j}\right) \cdot h\right]
$$

The application of this method is based on the graphical processing relation $\varphi(h)=\ln \left[J(h) / J_{0}\right]=$ $-\mu(E) \cdot h$, where $J_{0}$ is the intensity for $h=0$. The number of X-ray quanta with energies $E<E_{\max }$ passing through the filter with thickness $h_{\max }$ is negligible. We construct a tangent line $\varphi_{\tan }$ to the curve $\ln \left[J(h) / J_{0}\right]$ at the point $h_{\max }$. After that we produce subtraction $\varphi(h)-\varphi_{\tan }=\varphi_{1}$, which leads to a new curve without the contribution of $E_{\max }$. Then this procedure is repeated. Figure 4 shows the result of such process.

It should be noted that the bremsstrahlung as well as the characteristic radiation are presented at the recording range of the radiation. Therefore, the contribution of the characteristic radiation may lead to an error using the method of 'effective energy'. There are typical lines $K_{\alpha}$ of the radiation of the electrode materials $\mathrm{Cu}, \mathrm{Zn}$ and Fe in the recorded range, nevertheless their contribution to the total intensity of the spectrum is insignificant due to the small accelerating voltage $U$ used in the experiment $(U \leq 13 \mathrm{kV})$.

In the second series of measurements the discharge current was sustained with a capacitor of $C_{2}=0.011 \mu \mathrm{F}$ loaded up to $-13 \mathrm{kV}$ (Fig. 5). The level of the energy input into the discharge was decreased to the value of $E_{2} \sim 1 \mathrm{~J}$ and the magnitude of the discharge current reached $I_{r 2} \approx 2 \mathrm{kA}$. Other parameters were not changed.

The spectral composition of X-rays is shown in Fig. 5. The most intense radiation was observed for the electrode gap of $d=2 \div 3 \mathrm{~mm}$.

To control the temporal dependence of the discharge current a Rogowski coil was placed in the cathode circuit. The temporal characteristics of the X-ray emission were measured using a system consisting of two pin-photodiodes that were covered 
a)

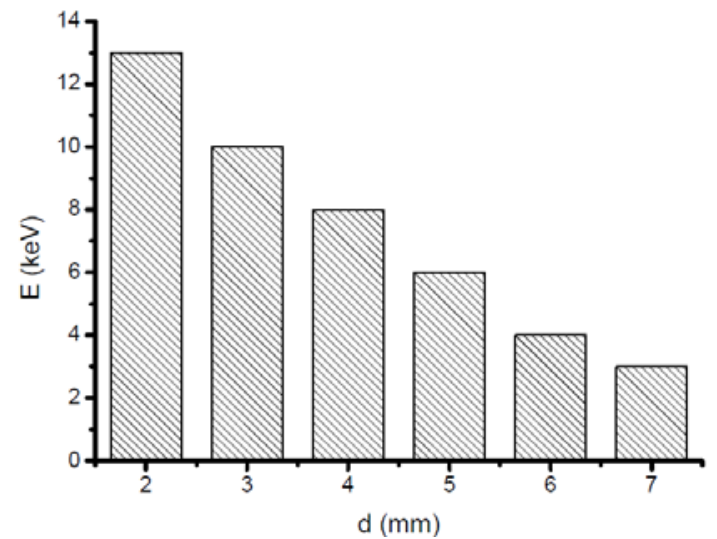

b)

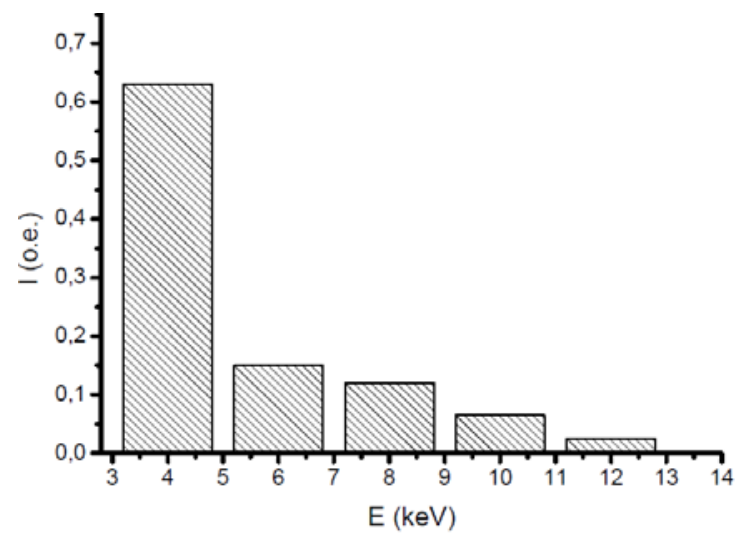

Fig. 5. Histograms of restoration of the X-ray spectrum for experimental conditions $C_{2} \approx 0.011 \mu \mathrm{F}, U=-13 \mathrm{kV}, E_{2} \sim$ $1 \mathrm{~J}: \mathrm{a})$ the average photon energy of the emitted X-ray spectrum as a function of the electrode gap size; b) the spectral composition of the X-ray source for the optimal gap size $d=2 \mathrm{~mm}$

a)

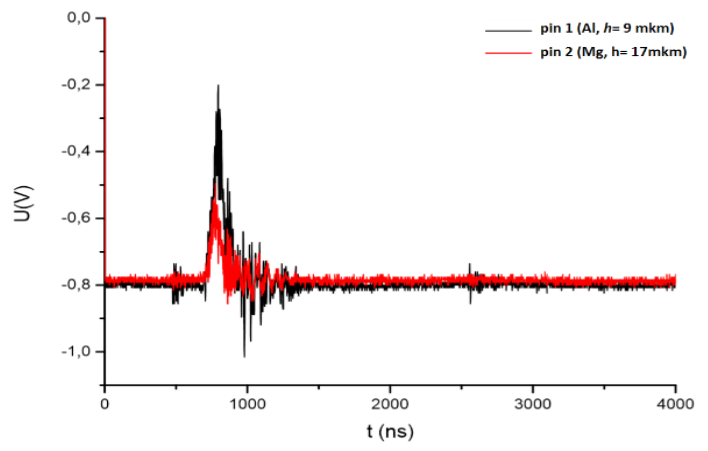

c)

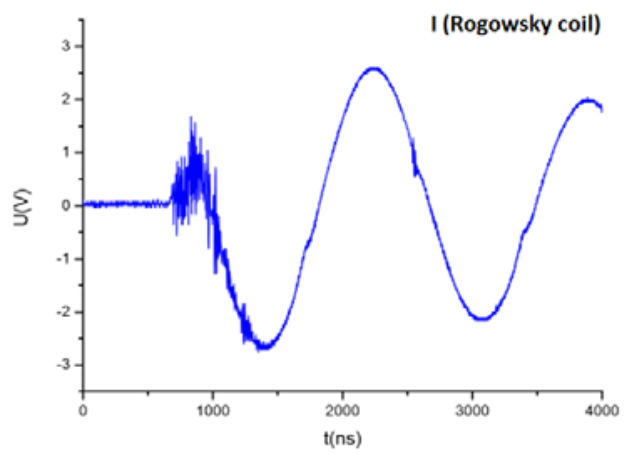

b)

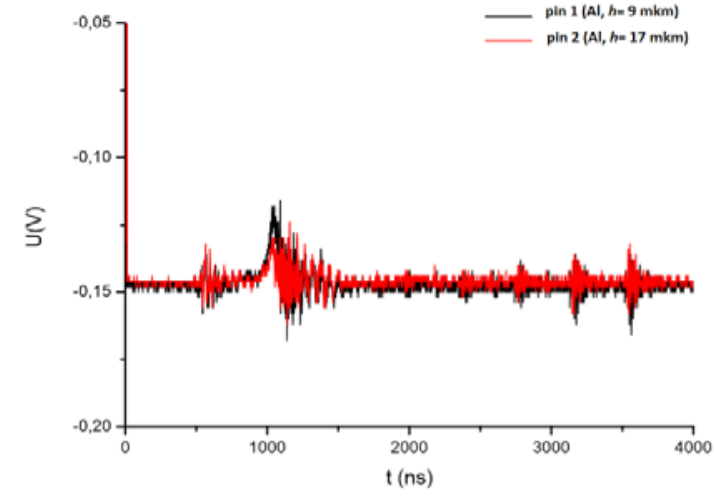

d)

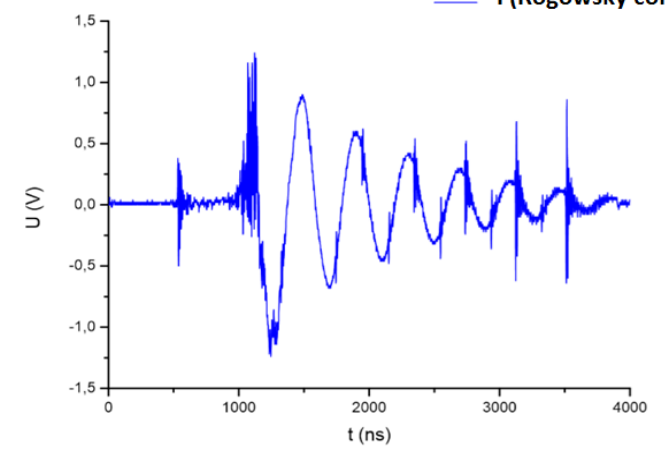

Fig. 6. The dynamics of the laser-triggering vacuum discharge: a) the signal from the pin-photodiodes, $C_{1}=0.22 \mu \mathrm{F}$, $\left.E_{1} \sim 17 \mathrm{~J} ; \mathrm{b}\right)$ the oscillogram of the discharge current, $\left.C_{1}=0.22 \mu \mathrm{F}, E_{1} \sim 17 \mathrm{~J} ; \mathrm{c}\right)$ the signal form the pin-photodiodes, $C_{2}=0.011 \mu \mathrm{F}, E_{2} \sim 1 \mathrm{~J} ; \mathrm{d}$ ) the oscillogram of the discharge current, $C_{2}=0.011 \mu \mathrm{F}, E_{2} \sim 1 \mathrm{~J}$.

with filters. The signals of the discharge current and the X-ray pulses were recorded by the digital oscilloscope Lecroy 'WaveAce2024' (Fig. 6).

It appears that moment of initiation of the discharge current oscillations correlated with the time when X-ray radiation was emitted. The discharge current shows damped oscillatory as the plasma was being developed. It should be noted that X-ray radiation was emitted in the initial stage of the discharge after $t \sim 300$ ns the following laser initiation pulse. The duration of the X-ray pulse was estimated as $t_{x r} \sim 100 \mathrm{~ns}$.

The results of numerically processed pin-photodiodes signals for different polarities and magnitudes of the voltage applied to the capacities $C_{1}$ and $C_{2}$ is shown in Fig. 7.

Figures $7 \mathrm{a}$ and $7 \mathrm{~b}$ show powerful nonlinear dependence of the X-ray emission in the investigated range of $1.3-1.6 \mathrm{keV}$ on the magnitude and polarity of the charging voltage. For $E_{1} \leq 1 \mathrm{~J}$ and the Fe anode the intensity of X-rays is 10 times weaker than with the reverse polarity. To interpolate the data shown in Fig. 7 an exponential function was used as the interpolating function. It was carried out disregarding the physical foundations.

In parallel with the X-ray studies the registration of the ion flux from plasma was performed via the TOF method $[10,11]$. Previously the composition of the electrode materials was investigated by the mass spectrum of ions generated in laser plasma only (Fig. 8a,b). This data was obtained with the mass spectra of laser-triggered vacuum discharge for a given current of the magnet (Fig. 8c,d).

Mass spectra of ions generated in the laser-triggered vacuum discharge were obtained for the case of Fe anode. For the Fe cathode we observed an 

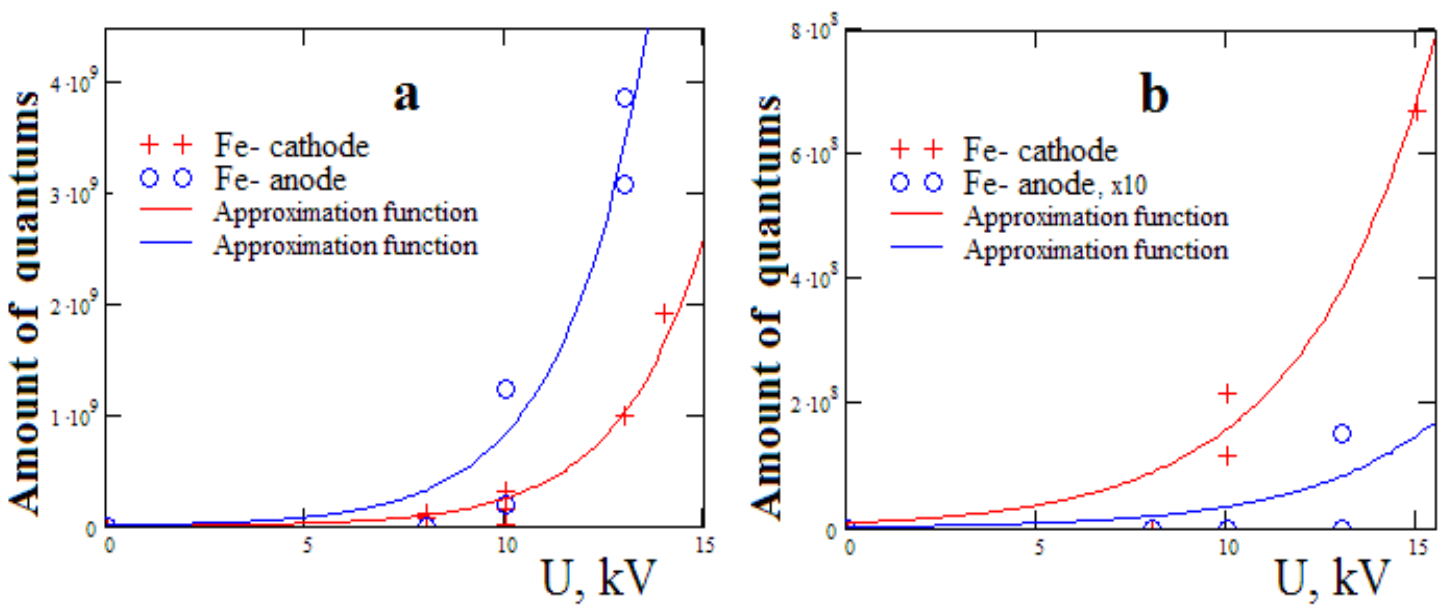

Fig. 7. The amount of X-ray quanta in the range of $1.3-1.6 \mathrm{keV}$ as a function of the applied amplitude and the sign of the power supply: a) $C_{1}=0.22 \mu \mathrm{F}, E_{1} \sim 17 \mathrm{~J}$; b) $C_{2}=0.011 \mu \mathrm{F}, E_{1} \sim 1 \mathrm{~J}$.

a)

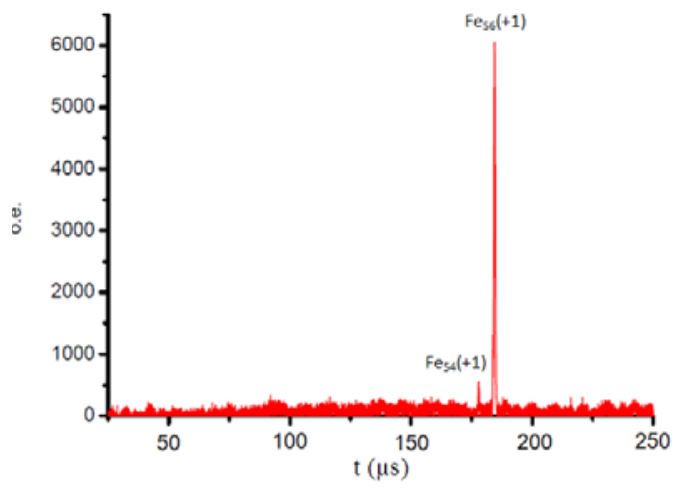

c)

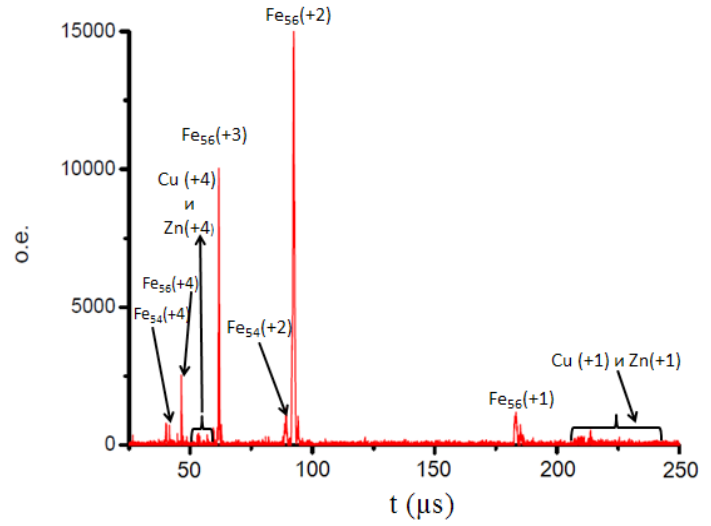

b)

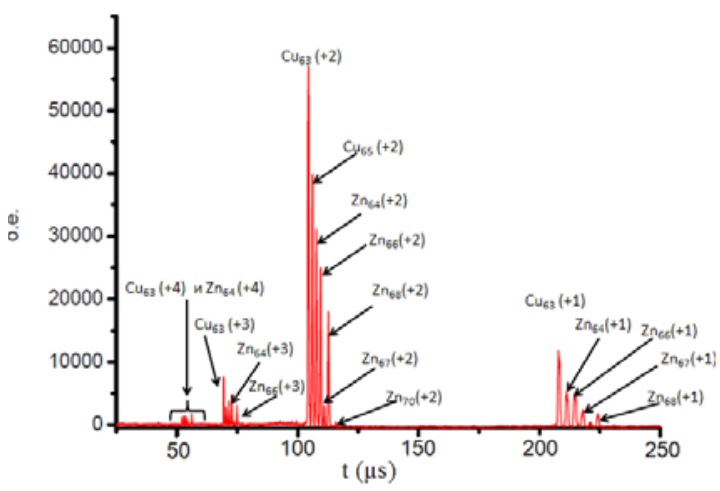

d)

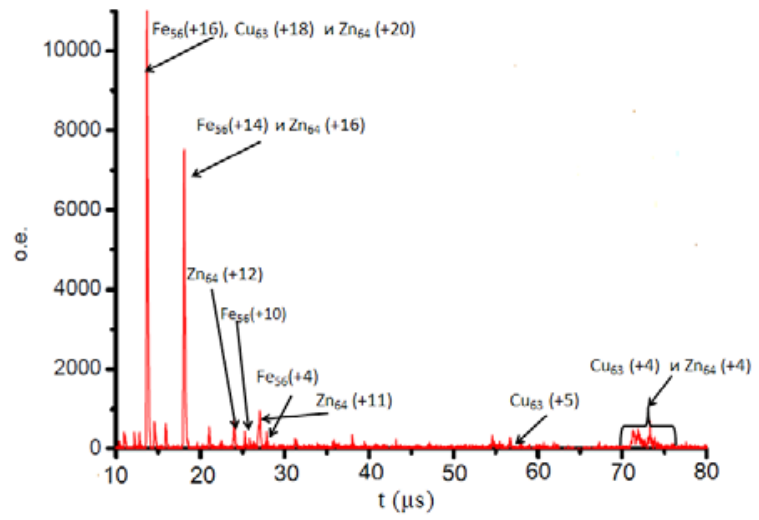

Fig. 8. Mass spectra of ions: a) the laser plasma (anode material); b) the laser plasma (cathode material); c) the laser-triggered plasma discharge with $C_{2}=0.011 \mu \mathrm{F}$ and the energy stored in the capacitor $E_{2} \approx 1 \mathrm{~J} ; \mathrm{d}$ ) the laser-triggered plasma discharge with $C_{1}=0.22 \mu \mathrm{F}$ and the energy stored in the capacitor $E_{1} \approx 17 \mathrm{~J}$.

extremely low yield of ions and therefore these mass spectra are not shown. The main contribution to the spectrum of multiply charged ions contribute zinc and iron ions, which is about two times more than copper ions. In the experiment we also assessed the number of carbon ions and oxygen ions, but the last one is negligible compared to the number of metal ions. General result for the charge states of ions of all energies is shown in Fig. 9a.

Figure $9 \mathrm{~b}$ shows the energy distribution of ions $\mathrm{Zn}^{(+21)}$ depending on the distance between the electrodes.

The ions of the electrode materials were predominantly observed in plasma. In the case of the stored energy $E_{1} \approx 17 \mathrm{~J}$ the average charge state of the ions was $\mathrm{Zn}^{(+21)}, \mathrm{Cu}^{(+20)}, \mathrm{Fe}^{(+18)}$ and the maximum charge state corresponded to $\mathrm{Zn}^{(+27)}, \mathrm{Cu}^{(+23)}$ and $\mathrm{Fe}^{(+21)}$, but the ion flux was two orders of magnitude higher than in the case of the stored energy $E_{2} \approx 1 \mathrm{~J}$, where the value of ion charge reached $\mathrm{Zn}^{(+6)}, \mathrm{Cu}^{(+5)}$ and $\mathrm{Fe}^{(+4)}$. Accordingly, when the stored energy was increased, the average value of ion charge in flux accrued [12].

According to [13], we can estimate the temperature of plasma in the initial hot stage. Namely, the presence in the plasma of ions with the charge number $Z$ with the ionization potential $\Delta$ allows us to speak about the value of the temperature in the generation of these ions $T_{e} \sim \Delta \times 0.2 \mathrm{eV} \mathrm{[14].} \mathrm{Ac-}$ cording to Fig. 8a plasma emitted a small amount of ions $\mathrm{Zn}^{(+27)}$ with the ionization potential $\Delta_{\mathrm{Zn}}=$ $2781 \mathrm{eV}$ [14]. Thus we obtain an upper bound on the plasma temperature: $T_{e} \sim 550 \mathrm{eV}$. 
a)

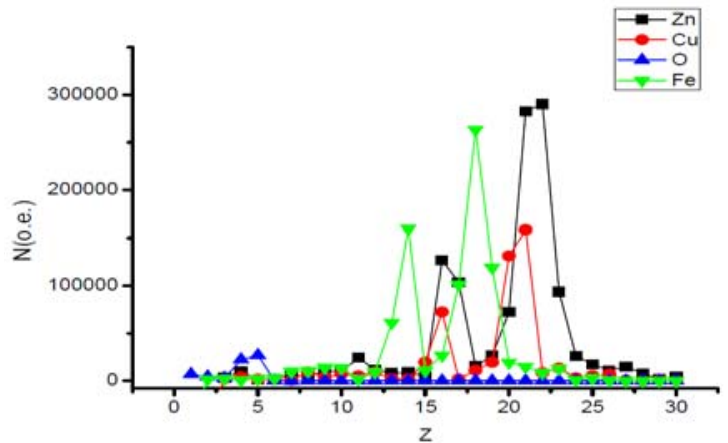

b)

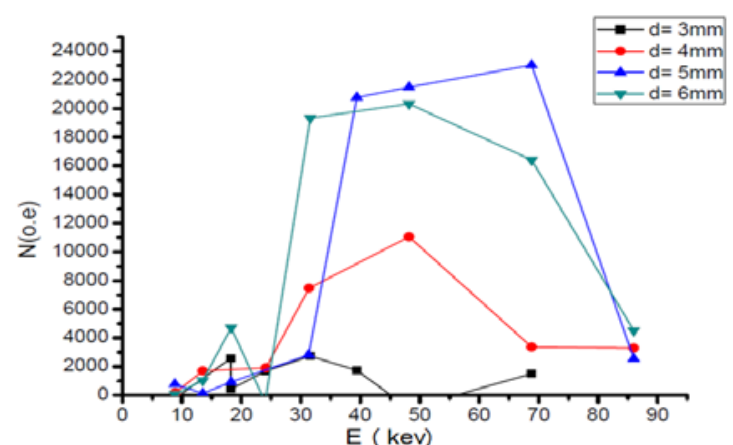

Fig. 9. a) The number of ions in the discharge, depending on their charge state $(d=5 \mathrm{~mm})$ and the discharge energy; $E \approx 17 \mathrm{~J}, C_{1}=0.22 \mu \mathrm{F} ; \mathrm{b}$ ) the number of ions $\mathrm{Zn}^{(+21)}$ and their energy depending on the distance between the electrodes.

Table 1. Characteristics of X-ray radiation depends on energy parameters of the electrode system

\begin{tabular}{ccc}
$\begin{array}{c}\text { Energy parameters } \\
\text { of the electrode system }\end{array}$ & $\begin{array}{c}\text { Total energy of X-rays, } \\
\mu \mathrm{J}(\mathrm{Fe} \text { anode) }\end{array}$ & $\begin{array}{c}\text { Total energy of X-rays, } \\
\mu \mathrm{J} \text { (Fe cathode) }\end{array}$ \\
\hline$C_{1}=0.22 \mu \mathrm{F}, E_{1} \sim 17 \mathrm{~J}$ & $\leq 400$ & $\leq 200$ \\
$C_{2}=0.011 \mu \mathrm{F}, E_{2} \sim 1 \mathrm{~J}$ & $\leq 2$ & $\sim 70$ \\
\hline
\end{tabular}

Using the estimate of the plasma temperature $T_{e}$ and the results of measurements with pin-photodiodes obtained above (Fig. 6a,b) we roughly estimated (the intensity of bremsstrahlung and recombination radiation $-I_{\varepsilon} \sim \exp (-\varepsilon / T e)$ ) the total energy of X-rays (in the range of $1-12 \mathrm{keV}$ ) in a pulse in the full solid angle $(4 \pi \mathrm{sr})$.

These data are shown in the Table 1.

\section{Conclusion}

It is important to note that the highest intensity of the $\mathrm{X}$-ray emission, the maximal number and the charge state of ions were observed at the same distance: $d=5 \div 6 \mathrm{~mm}$ for $C_{1}=0.22 \mu \mathrm{F}$ and $d=2 \div 3 \mathrm{~mm}$ for $C_{2}=0.011 \mu \mathrm{F}$. In the case of stored energy $E_{1} \approx$ $17 \mathrm{~J}$ the highest X-ray emission was observed when $d \approx 5 \mathrm{~mm}$ and the spectral composition consisted essentially of hard component of radiation. But in the case of $E_{2} \approx 1 \mathrm{~J}$ the maximal intensity was obtained when $d=2 \div 3 \mathrm{~mm}$. The possibility to adjust the X-ray spectral composition by changing the geometry of the electrode system was demonstrated. The information about the composition of the laser-induced vacuum discharge plasma was obtained. The mass spectrum of charged ions was measured up to $\mathrm{Zn}^{(+21)}, \mathrm{Cu}^{(+20)}$ and $\mathrm{Fe}^{(+18)}$ inclusively.

Apparently if we know the charge spectrum of the plasma ions (see Fig. 8), we can estimate or simulate the characteristics of bremsstrahlung and recombination X-ray. In the mass spectrum the emission of a large number of high-energy Ne-like ions $\mathrm{Zn}^{(+20)}, \mathrm{Cu}^{(+19)}$ and $\mathrm{Fe}^{(+16)}$ (Fig. 8) was also seen, which could be a source of intense stimulated $\mathrm{X}$-ray radiation in plasma $[10,11]$. This reveals new possibilities in the study of laser-discharge plasma as a stable regulated power of X-ray radiation, and a source of beams of high-energy ions, which can significantly extend the range of application of the discharge plasma with laser triggering for applications in science and technology.

\section{References}

1. Korop, E. D., Meyerowitz, B. E., Sidel'nikov, J. V., \& Sukhorukov, S. T. (1979). Micropinches in high-current diode. Sov. Phys. Usp., 129(1), 87-112.

2. Georgescu, N., Serbanescu, C. G., \& Sandolache, G. (2001). Reproducibility conditions for the pulsed $\mathrm{X}$-ray emission in a vacuum spark discharge. Nukleonika, 46(Suppl. 1), 17-20.

3. Korobkin, Yu. V., Paperny, V. L., Romanov, I. V., Rupasov, A. A., \& Shikanov, A. S. (2008). Micropinches in laser induced moderate power vacuum discharge. Plasma Phys. Control. Fusion, 50, 1-14, DOI: 10.1088/0741-3335/50/6/065002.

4. Korobkin, Yu. V., Romanov, I. V., Rupasov, A. A., \& Shikanov, A. S. (2005). Vacuum discharge instability in laser initiation of the cathode spot. J. Tech. Phys., 75(9), 34-39.

5. Baechler, S., Monnin, P., Aroua, A., Valley, J. F., Perrier, M., Trueb, P., \& Verdun, F. R. (2010). Exposure in dental radiology: A comparison between intra-oral, panoramic and tomographic examinations. Radiat. Prot. Dosim., 139(1/3), 303-305.

6. Bashutin, O. A., Alkhimova, M. A., Vovchenko, E. D., Dodulad, E. I., Savelov, A. S., \& Sarancev, S. A. (2013). Effect of electrode system on the radiative characteristics of the vacuum spark. Plasma Phys., 39(11), 1006-1016.

7. Basov, N. G., Zaharenko, Yu. A., Rupasov, A. A., Slizkov, V. G., \& Shikanov, A. S. (1989). The diagnostics of the dense plasma (pp. 127-130). Moscow: Nauka.

8. Averin, M. S., Bajkov, A. Y., Bashutin, O. A., Vovchenko, E. D., \& Savelov, A. S. (2006). Estimates of the electron temperature of the plasma discharge to weaken micropinch X-ray flux in the emulsion. Instrum. Exp. Tech., 2(1), 5.

9. Dolgov, A. N., Savelov, A. S., \& Salakhutdinov, G. Kh. (2008). Application of a spectrometer complex of equipment for x-ray diagnostics of plasma of pulse installations. Prikladnaya Fizika (Applied Physics), 5, 35-40.

10. Bykovskii, Y. A., \& Nevolin, V. N. (1985). Laser mass spectrometry. Moscow: Energoatomizdat.

11. Basov, N. G., Zakharenkov, Y. A., Rupasov, A. A., Sklizkov, G. V., \& Shikanov, A. C. (1989). Diagnosis of a dense plasma. Moscow: Nauka. 
12. Romanov, I. V., Paperny, V. L., Korobkin, V., Kiselev, N. G., Rusape, A. A., \& Shikanov, A. S. (2013). Effect of laser pulse parameters on characteristic source of multiply charged ions of metals based on laser-induced spark average power. Lett. Tech. Phys., $39(8), 62-70$.
13. Bufetov, I. A., Bufetova, G. A., Kravtsov, S. B., Fyodorov, V. B., \& Fomin, V. K. (1995). Heating of the plasma on a metal target by nanosecond pulses of the first, second and fourth harmonics Nd-laser. Quantum Electron., 25(8), 794-798.

14. http://physics.nist.gov/PhysRefData/ASD/levels_form.html. 